\title{
Bose-Einstein Condensation
}

Atoms are normally considered as particles but, according to quantum mechanics, they also have wave-like properties. Indeed, an atom has an equivalent wavelength, the deBroglie wavelength, which is inversely proportional to the atom's momentum. As atoms are cooled they slow down and their deBroglie wavelength increases. Satyendra Bose and Albert Einstein predicted 70 years ago that at a low enough temperature the wavelength exceeds the interparticle spacing and the atoms begin to overlap. The atoms become indistinguishable, effectively entering - by a process called Bose-Einstein condensation (BEC) - a coherent state where the laws of quantum mechanics govern the behaviour of the macroscopic system. BEC has been observed in superfluid helium -4 and superconductivity, both being states of matter in which bosons (integral-spin particles) condense into macroscopic quantum states. But the bosons in these systems interact with each other, so to better understand BEC, physicists have tried for sometime to bring about condensation in an ideal gas of noninteracting atoms.

\section{The JILA Experiment}

The JILA experiment consists of three stages: A - optical loading, (pre)cooling \& polarization; B - magnetic trapping \& evaporative cooling in the absence of light; $\mathrm{C}$ - optical detection.

$A$ - laser cooling stage. For $300 \mathrm{~s}$, about $10^{7}$ atoms are gathered from the low-velocity tail of a room-temperature Rb-vapour at some $10^{-11}$ mbar into a so-called "dark" magneto-optical trap (MOT), which is most efficient in collecting a large number of atoms by optical forces. The gas is rapidly (pre)cooled in the MOT to about $20 \mu \mathrm{K}$ and subsequently magnetically polarized in a small magnetic bias field by optical pumping.

$B$ - trapping and cooling. Polarized atoms are magnetically trapped in a rapidly applied timeorbiting potential (TOP) trap, which consists of a set of coils in anti-Helmholtz configuration with a small, uniform transverse field, rotating at $7.5 \mathrm{kHz}$, superimposed. The TOP provides an effectively harmonic potential with an axial frequency of about $120 \mathrm{~Hz}$ and radial frequency of about $42 \mathrm{~Hz}$. In the TOP, the gas is adiabatically compressed to reach the starting conditions for evaporative cooling at about $90 \mu \mathrm{K}$ with a number density of about $2 \times 10^{10} \mathrm{~cm}^{-3}$, sufficiently large to have the elastic collision rate dominate over the loss rate in the gas. The evaporation takes $70 \mathrm{~s}$ and BEC is achieved with $2 \times 10^{4}$ atoms at approximately $170 \mathrm{nK}$. The evaporation is induced by a RF transition to an untrapped state of the atoms at the edge of the sample.

$C$ - optical detection. At the start of the detection stage the trap is expanded adiabatically to a larger size to allow a fast (destructive) absorption measurement in which the sample is imaged on a CCD camera. The appearance of a diffraction ring marks the growth of a partially resolved structure in the sample. This is consistent with BEC in an inhomogeneous sample, where the condensate is expected to appear as a very small, dense gas cloud at the centre of the potential well. To confirm this interpretation, the momentum distribution in the sample was measured by suddenly switching-off the trap and observing the expanding gas cloud. In this process both isotropic expansion of the (thermal) non-condensate fraction was observed as well as an anisotropic expansion, as is to be expected for a suddenly released anisotropic single quantum state.

\section{INELASTIC X-RAY SCATTERING}

\section{Great Potential Demonstrated}

\section{F. Sette and G. Ruocco describe why the confirmation, at the European Synchro- tron Radiation Facility, of fast sound in water at momentum transfers of from 4 to $14 \mathrm{~nm}^{-1}$ using inelastic $X$-ray scattering with a resolution in the meV range demonstrates the technique's important capabilities.}

The determination of the collective dynamics in liquid water is a long-standing problem which dates back to 1974 , when Rahman and Stillinger [1] proposed the existence of high-frequency collective excitations (so-called fast sound) propagating with a velocity which is much higher than that of ordinary sound. The proposal stimulated various studies, justified not only by the practical importance of liquid-like water but also by the implications associated with the possible coexistence of different kinds of acoustic-like excitations in molecular liquids.

F. Sette heads the Inelastic X-Ray Scattering Group in the Experiments Division, European Synchrotron Radiation Facility, BP 220, F-38043 Grenoble Cedex.

G. Ruocco is with the Università di L'Aquila and the Istituto Nazionale di Fisica della Materia, 1-67100 L'Aquila dilute, with only a few collisions per atom per second. Since, in the absence of interactions, the compressibility of a Bose condensate is infinite, the condensate will be compressed by the trapping potential until the interatomic interactions counterbalance the potential, thus affecting the size and shape of the condensate. This effect has also been observed at JILA (see figure).

Many intriguing problems await experimental investigation. Most prominent among them are the relation between $B E C$ and superfluidity, the kinetics of BEC, the size and number dependence of the transition, optical properties, dimensionality aspects, the interaction between two condensates, the use of the condensate as a source for a coherent atomic beam, and the differences with respect to Fermi systems. One controversial topic - the stability of condensates under an attractive mean field (negative scattering amplitude) - is already being addressed by Randall Hulet, who observed diffraction rings indicating an internal structure in ultra-cold samples of $7 \mathrm{Li}$. Similar experiments can be done with ${ }^{85} \mathrm{Rb}$. There are suggestions that the scattering amplitude of the heavy alkalis can be modified and its sign possibly even changed. Clearly, an exciting period lies ahead. It is fortunate that the results obtained by the group of Cornell and Wieman were realized with methods accessible to many groups in university environments as this will allow a wide exploration of the fascinating Bose-Einstein condensates.

claimed that there was no fast sound in heavy water.

\section{Inelastic Scattering at the ESRF}

The dynamical structure factor in liquid water has recently been determined by inelastic $\mathrm{X}$-ray scattering at a total energy resolution of $3.2 \mathrm{meV}$ using a newly constructed instrument at the European Synchrotron Radiation Facility, Grenoble. This technique, besides offering the possibility to investigate a much larger $q-\omega$ region than for neutrons, presents other important advantages with respect to neutrons for the specific problem of sound propagation in water. They are: a) the absence of an incoherent contribution to scattering spectra; and b) the possibility to study normal (as opposed to heavy) water using direct measurements.

The new results strongly favour theoretical models for the dynamics of water which account for fast sound in terms of an anomalously large increase at high frequencies of the speed of propagation of collective acoustic-like excitations $[4,5]$. The physical origin of this increase lies mostly in the interplay between the different contributions to the intermolecular potential, i.e., the different roles played by electrostatic and Lennard-Jones-like terms in determining the
Moreover, in 1995 Bermejo et al. [6] made new neutron scattering measurements and tried to clarify the origin of these excitations [3-5]. Despite these efforts, the issue could not be settled, mainly because neutron data were only available for a limited region of momentum transfer - frequency $(q-\omega)$ space 

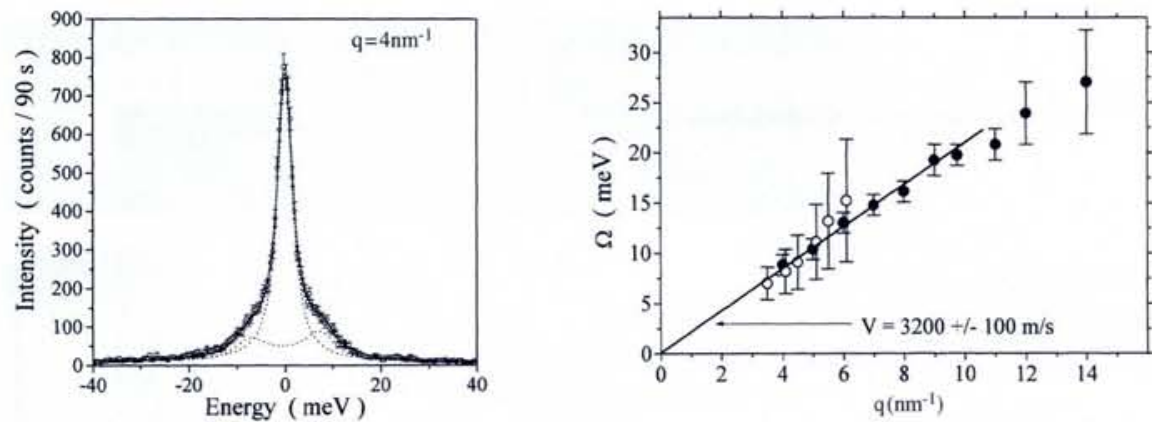

Fig. 1 - Inelastic X-ray scattering from water. a, left) A scattering spectrum for water taken at momentum transfer $q$ of $4 \mathrm{~nm}^{-1}$. The experimental data are superimposed on the fitted curve (solid line). The contributions of the central peak and the side peaks from the two terms in Eq. 1 are shown as dotted curves.

$b$, right) The dispersion relation (central frequency as a function of the momentum transfer) for collective excitations in water. $\bullet$ : inelastic $x$-ray scattering data for $\mathrm{H}_{2} \mathrm{O}$ as obtained from fitted spectra; O: inelastic neutron scattering data for $\mathrm{D}_{2} \mathrm{O}$ (from [2]). The gradient of the solid line corresponding to the best linear fit to the $X$-ray data up to $10 \mathrm{~nm}^{-1}$ gives a speed of sound of $3200 \pm 100 \mathrm{~m} / \mathrm{s}$. intermolecular distances and the $q-\omega$ dependence of the molecular dynamics [5].

The inelastic $\mathrm{X}$-ray scattering spectra were measured at $q$-values of $4-14 \mathrm{~nm}^{-1}$ energy resolution of $3.2 \mathrm{meV}$ [7]. Fig. 1a gives, as a typical example, a spectrum taken at $q=4 \mathrm{~nm}^{-1}$ together with the corresponding fitted curve. In order to determine the energy positions and the damping of excitations at each $q$-value, the spectra were modelled using a function $F(q, \omega)$ consisting of a Lorentzian for the central peak and a damped oscillator [8] for the two side peaks:

$$
\begin{aligned}
F(q, \omega)= & I_{0}(q) \frac{\Gamma_{0}(q)^{2}}{\Gamma_{0}(q)^{2}+\omega^{2}}+ \\
& {[n \omega+1] l(q) \frac{\omega \Gamma(q)^{2} \Omega(q)}{\left\{\Omega(q)^{2}-\omega^{2}\right\}^{2}+\Gamma(q)^{2} \omega^{2}}(1) }
\end{aligned}
$$

fast-sound modes propagate in an atomic network constituted primarily by the hydrogen atoms. The data indicate instead that fast sound involves the whole molecule (the isotopic shift expected for the whole molecule is approximately $5 \%$, well within the error bars, while it would be approximately $40 \%$ if individual hydrogen atoms were involved).

This determination of collective excitations in liquid water demonstrates the ability of inelastic X-ray scattering at meV energy resolutions to study the dynamics of disordered systems. Propagating collective excitations have been found at momentum transfers of $4-14 \mathrm{~nm}^{-1}$, and the existence of fast sound has been established over this entire momentum range. using X-rays of $17794 \mathrm{eV}$ with an overall
It is worth mentioning that the technique may also find important applications in studies where only small sample volumes are available. Taking advantage of the small $\mathrm{X}$-ray beam size of $300 \times 300 \mu \mathrm{m}^{2}$, one can also investigate the dynamics of small exotic crystals, and of various materials under extreme thermodynamic conditions such as those present at very high pressure and high temperatures.

[1] Rahman A. \& Stllinger F.H., Phys. Rev. A 10 (1974) 368.

[2] Teixeira J., Bellissant-Funel M.C., Chen S.H. \& Dorner B, Phys. Rev. Lett. 54 (1985) 2681.

[3] Impey R.W., Madden P.A. \& McDonald I.R., Mol. Phys. 46 (1982) 513; Ricci M.A., Phys. Rev. Lett. 61 (1988)1958; Ricci M.A et al., Phys. Rev. A 40 (1989) 7226; Sastry S., Sciortino F. \& Stanley E., J. Chem. Phys. 95 (1991) 7775; Balucani U. et al., Chem. Phys. Lett. 209 (1993) 408.

[4] Wojcik W. \& Clementi E., J. Chem. Phys. 85 (1986) 6085

[5] Balucani U. et al., Phys. Rev. E 47 (1993) 1677; Sciortino F. \& Sastry S., J. Chem. Phys. 100 (1994) 3881.

[6] Bermejo F.J. et al., Phys. Rev. E 51 (1995) 2260.

[7] Sette F. et al., Phys. Rev. Lett. 75 (1995).

[8] Fak B. \& Dorner B., ILL Tech. Report No. 92FA008S (1992).
Here $\Gamma_{0}(q)$ and $I_{0}(q)$ are the width and intensity, respectively, of the central peak, $\Gamma(q)$ and $I(q)$ are the damping and intensity of the side peaks with a central frequency $\Omega(q)$, and $n(\omega)$ is the Bose factor. This specific function was chosen to allow a direct comparison with the previous neutron data $[2,6]$, which had been analyzed using the same model. The convolution of $F(q, \omega)$ with a Lorentzian representing the resolution function was fitted to the experimental data, after subtraction of the detector's dark counts.

The derived values of $\Omega(q)$ are shown in Fig. $1 \mathrm{~b}$ together with the analogous quantities obtained by TBCD from their neutron data. The $\Omega(q)$ values follow a linear behaviour characterized by a speed of sound of $3200 \pm 100 \mathrm{~ms}^{-1}$ up to $q=10 \mathrm{~nm}^{-1}$ (for higher $q$-values there is an indication of a decrease in the velocity). This value of the velocity of sound is identical, within the experimental error, to that of $3300 \pm 250$ $\mathrm{ms}^{-1}$ reported by TBCD for heavy water.

\section{Firm Conclusions}

The results shown in Fig. $1 \mathrm{~b}$ and their comparison with those of TCBD allow for two firm conclusions: a) fast sound does indeed exist in water in the $q$-region between 4 and $14 \mathrm{~nm}^{-1}$, and b) the same value for the velocity of fast sound in $\mathrm{H}_{2} \mathrm{O}$ and $\mathrm{D}_{2} \mathrm{O}$ rules out the possibility that the

\section{FELIX USER FACILITY}

\section{CALL FOR PROPOSALS}

DEADLINE: 1 October 1995

The international free-electron laser user-facility FELIX provides intense ( $\$ 20 \mathrm{MW}$ in a (sub) ps-pulse), continuously-tuneable in the infrared spectral range (5-110 $\mu \mathrm{m})$. Those interested in the use of FELIX in the period January - June 1996 are invited to submit a research proposal before 1 October 1995 . The proposal will be reviewed by a Programme Advisory Committee, in order to establish a priority rating. The use of FELIX is free of charge for researchers from the academic community.

An information package about FELIX and ancillary equipment, including guidelines for submitting a proposal, is available from Mrs. Laura M.P. van Veenendaal, Secretary of Laser Physics Department, FOM-Institute for Plasma Physics "Rijnhuizen", Nieuwegein, The Netherlands.

Requests should preferably be made by fax: $+31-3402-31204$ or by e-mail: lauravV@ rijnh.nl.

\section{GRENOBLE HIGH MAGNETIC FIELD LABORATORY POST-DOCTORAL RESEARCH POSITIONS}

Applications are invited for post-doctoral research fellowships to participate, in different areas of condensed matter physics, in the experimental investigation of magnetic field dependent properties. The positions, available immediately for qualified scientists, are funded by the European Community, for at least one year and up to two years.

The scientists will be integrated into different groups to develop specific projects on magnetism, high- $T_{\mathrm{c}}$ superconductivity, metal physics, and semiconductor physics. They will be also partly in charge of training and assisting the users of the High Magnetic Field Facility.

Applicants should have a Ph.D. with a good training in experimental investigations including transport, optical measurements performed at low temperatures.

Applicants should be nationals of European Union countries (except France). They should send by 1 October 1995 a full CV with names and addresses of two referees to:

Dr. G. Martinez, Grenoble High Magnetic Field Laboratory, MPIF/CNRS, 25 avenue des Martyrs, F-38042 Grenoble Cedex 09, France. 